\title{
Clinical analysis of 13 males with primary choriocarcinoma and review of the literature
}

This article was published in the following Dove Press journal:

OncoTargets and Therapy

20 June 2014

Number of times this article has been viewed

\author{
Fang Jiang' \\ Yang Xiang' \\ Feng-Zhi Feng' \\ Tong Ren' \\ Zhu-Mei Cui ${ }^{2}$ \\ Xi-Run Wan' \\ 'Department of Obstetrics and \\ Gynecology, Peking Union Medical \\ College Hospital, Peking Union \\ Medical College, Chinese Academy \\ of Medical Sciences, Beijing, People's \\ Republic of China; ${ }^{2}$ Department \\ of Obstetrics and Gynecology, the \\ Affiliated Hospital of the Medical \\ College, Qingdao University, Qingdao, \\ People's Republic of China
}

Objective: To analyze the management and prognosis of primary choriocarcinoma (PCC) in male patients.

Methods: The clinical records of males with PCC who were treated at Peking Union Medical College Hospital between 1990 and 2012 were analyzed retrospectively. The literature regarding this clinical condition was also reviewed.

Results: The median survival interval of the 13 patients treated at Peking Union Medical College Hospital was 54 months (range, 6-115 months), and the 1- and 3-year survival rates were 53.8\% and $43.1 \%$, respectively. All patients were treated with surgery; 12 were treated with combined chemotherapy. After including 100 cases found in the literature, for a total of 113 patients, the median survival interval was 10 months (range, 6.4-13.6 months). The testis was the most common primary site (36.2\%). Most patients $(70.9 \%)$ had metastatic lesions at diagnosis. Univariate and multivariate analyses revealed that longer median overall survival was significantly associated with patient age $<34$ years old ( 48 months vs 10 months, odds ratio [OR] $=0.47, P=0.029$ ), the presence of other histological components (54 months vs 11 months, $\mathrm{OR}=0.54, P=0.011$ ), and combined chemotherapy and surgical treatments ( 14 months vs 2.5 months, $\mathrm{OR}=0.18, P=0.002$ ).

Conclusion: PCC is an extremely rare disease among men, and its prognosis is much worse than that of gestational choriocarcinoma. The complete resection of the primary site and metastases followed by chemotherapy seems to provide patients with the best chance at survival. Furthermore, additional chemotherapy cycles might facilitate better progress.

Keywords: PCC, men, prognosis

\section{Introduction}

Primary choriocarcinoma (PCC) is a rare malignancy that can occur in both men and women. In men, the most common primary site is the testis. ${ }^{1}$ When the primary tumor is extragonadal, it can occur in the mediastinum, ${ }^{2}$ retroperitoneum, ${ }^{3}$ lung, ${ }^{4}$ brain, ${ }^{5}$ or digestive tract. ${ }^{6}$ Because its clinical symptoms are atypical, early diagnosis is difficult. In contrast to gestational choriocarcinoma, the treatment and prognosis of PCC are poor, especially in males. The clinical behavior, tumor characteristics, and prognostic parameters of PCC have not been clearly described. We retrospectively analyzed cases of males with PCC treated at Peking Union Medical College Hospital (PUMCH) between 1990 and 2012. Furthermore, we reviewed the relevant literature to increase the understanding of this disease.

\section{Materials and methods}

\section{Study design}

The purpose of this retrospective study is to analyze the management and prognosis of PCC in male patients. All case records and images were reviewed, with follow-up 
assessments extending to March 2013. All living patients were clinically interviewed. Patient death information was acquired from census data, and the literature was reviewed simultaneously. Death due to choriocarcinoma was the sole endpoint considered in this study.

\section{Study population}

Inclusion criteria: Males with PCC who were treated at PUMCH between 1990 and 2012. All patients were diagnosed pathologically. The cases reviewed were indexed in PubMed from the English-language literature from 1990 to 2012. Exclusion criteria: cases without complete descriptions of the treatment and prognosis were excluded.

\section{Statistical analysis}

Statistical analysis was performed with SPSS 17.0 statistical software. Baseline characteristics are described using the median values. The $t$-test or Mann-Whitney $U$ test was used for continuous variables, and the chi-square test or Fisher's exact tests was used for categorical variables. Univariate analyses of survival were performed using the Kaplan-Meier method, and the evaluation of differences between the two groups was performed using the log-rank test. The following variables were assessed as potential prognostic factors with respect to overall survival (OS) in univariate analyses: age, metastasis, preoperative diagnosis, the presence of chemotherapy, histology, and surgery. Results with $P$-values $<0.05$ were considered significant. The Cox proportional hazards model was used to calculate the hazard ratios and 95\% confidence intervals (CIs) in the univariate analyses. The aforementioned variables were considered potential prognostic factors if $P<0.1$.

\section{Results}

\section{General conditions}

A total of 3,633 cases of gestational neoplasia and 46 cases of PCC at PUMCH were reported between 1990 and 2012, for a ratio of $79: 1$. Of the patients with $\mathrm{PCC}, 13$ were male and 33 were female (ratio $=1: 12.5$ ). A total of 418 male patients with other germ cell tumors were identified over the same period, for a ratio of 1:33. The clinicopathological data and follow-up information of the 13 patients at PUMCH are presented in Table 1. The median age of these patients was 31.0 years old (range, 52 days -55 years).

In addition, a total of 100 males with PCC with complete OS data have been documented previously across 82 articles published between 1990 and 2012. These 100 cases were combined with the 13 cases treated at PUMCH and included in the analysis (Table 2). The median age of all males with choriocarcinoma was 34 years old (range, neonate to 80 years old). The most common age range was 20-29 years old, followed by $30-39$ years old.

\section{Lesion sites}

Seven primary sites were identified among the males at PUMCH, including the testis (four cases), mediastinum (three cases), pineal body (two cases), lung (one case), retroperitoneum (one case), jejunum (one case), and stomach (one case). The lung was the most common metastatic site in $53.8 \%(7 / 13)$ of the sample, followed by the liver, brain, retroperitoneum, and bone. Furthermore, the testis was the most common primary site for all 113 cases at $36.2 \%$ $(41 / 113)$, followed by the mediastinum (14 cases) and stomach (13 cases).

\section{Histology}

Eight cases at PUMCH were pure choriocarcinoma, and the others were mixed germ cell tumor (including mature teratoma, immature teratoma, and embryonal carcinoma) and PCC. Including the literature cases, pure choriocarcinoma (89/113) was the most common pathological type.

\section{Treatment}

All patients were treated with surgery. The primary sites of ten patients were totally resected. Of these ten patients, three did not show metastasis; seven patients had metastases, and only two of these patients had their metastatic lesions excised. One patient had his primary site partially resected, and only the metastatic lesions were excised from another two patients. Twelve patients were treated with combined chemotherapy (Table 1). The median number of cycles was seven (range, 3-27 cycles). The major drugs used for treatment included actinomycin, etoposide, methotrexate, cyclophosphamide, cisplatin, and bleomycin. A patient with a case of primary testicular choriocarcinoma with a mature teratoma component did not receive chemotherapy after surgery because his $\beta$-hCG dropped to a normal level immediately after surgery. Three patients received radiotherapy (Cases 8-10; see Table 1).

\section{Follow-up, overall survival, and prognostic factors}

For PUMCH cases (Table 1), the median survival interval was 54 months (Figure 1). The 1- and 3-year survival rates were $53.8 \%$ and $43.1 \%$, respectively. Only three patients had preoperative diagnoses. Three types of clinical outcomes 


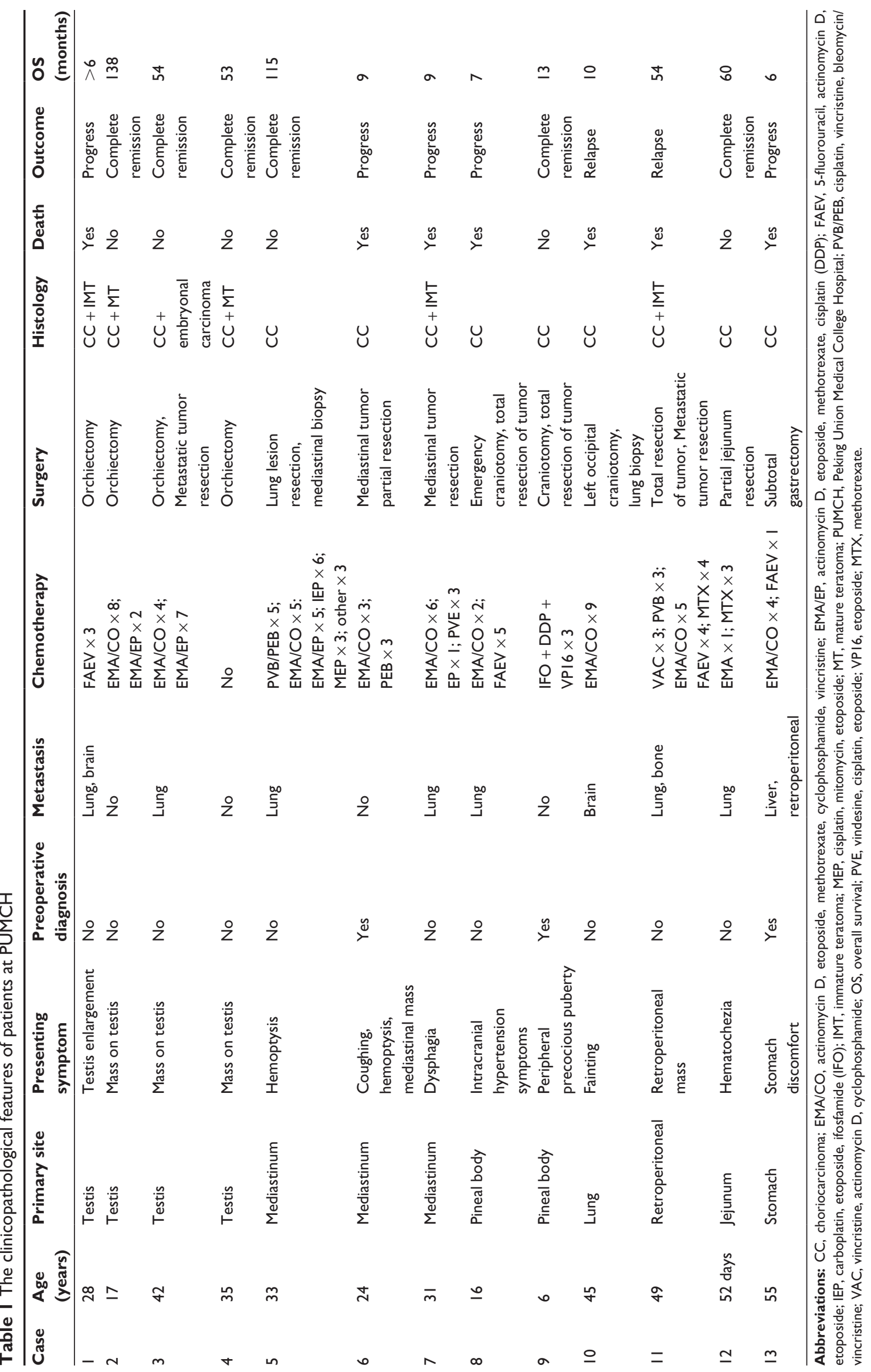


Table 2 A summary of the PCC cases reported in the literature

\begin{tabular}{|c|c|c|c|}
\hline \multirow[t]{4}{*}{ Primary site } & \multicolumn{3}{|c|}{ Age (years) } \\
\hline & \multicolumn{3}{|c|}{$\begin{array}{l}\text { Median, } 34 \text { (range, neonate to } \\
80 \text { years old) }\end{array}$} \\
\hline & Cases (\%) & PUMCH & All (\%) \\
\hline & $N=100$ & $N=13$ & $N=1 / 3$ \\
\hline Testis & $37(37)$ & 4 & $41(36.2)$ \\
\hline Mediastinum & II (II) & 3 & $14(12.4)$ \\
\hline Stomach & $12(11)$ & I & $13(11.5)$ \\
\hline Liver & $12(12)$ & 0 & $12(10.6)$ \\
\hline Lungs & $10(10)$ & I & II (9.7) \\
\hline Brain & $6(6)$ & 2 & $8(7.1)$ \\
\hline Small intestine & $6(6)$ & 0 & $6(5.3)$ \\
\hline Other & $6(6)$ & 2 & $8(7.1)$ \\
\hline \multicolumn{4}{|l|}{ Treatment } \\
\hline None & 14 & 0 & 14 \\
\hline Chemotherapy & 29 & 0 & 29 \\
\hline Surgery & 4 & I & 5 \\
\hline Chemotherapy + radiotherapy & 8 & 0 & 8 \\
\hline Surgery + chemotherapy & 42 & 9 & 51 \\
\hline $\begin{array}{l}\text { Surgery + chemotherapy }+ \\
\text { radiotherapy }\end{array}$ & 3 & 3 & 6 \\
\hline \multicolumn{4}{|l|}{ Histology } \\
\hline Pure & $8 I(8 I)$ & 8 & $89(78.8)$ \\
\hline Combined with other component & $19(19)$ & 5 & $24(2 \mid .2)$ \\
\hline
\end{tabular}

Abbreviations: PCC, primary choriocarcinoma; PUMCH, Peking Union Medical College Hospital.

were observed. 1) Complete remission: Six patients survived without recurrence (follow-up time: 21-138 months). Three patients $(50 \%)$ had metastases at the initial diagnosis.

2) Progressive disease: Five patients died from progressive disease within 13 months. Four patients (80\%) had metastases at the initial diagnosis. All patients were treated with intensive chemotherapy, and three were diagnosed preoperatively via the detection of elevated serum $\beta$-hCG. 3) Relapse (two patients): a 45-year-old man suffered a stroke,

A

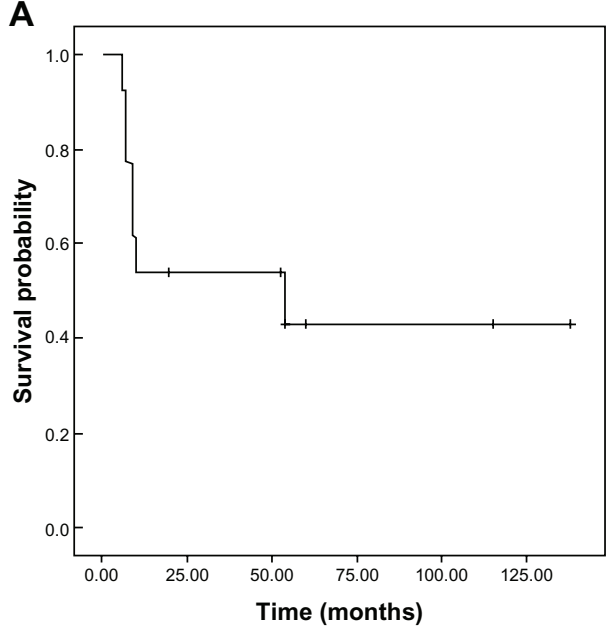

Figure I OS curves: (A) I 3 PUMCH cases; (B) all II 3 cases.

Abbreviations: OS, overall survival; PUMCH, Peking Union Medical College Hospital. and an emergency craniotomy operation was performed. The histological finding was metastatic choriocarcinoma. A postoperative lung biopsy confirmed the presence of primary pulmonary choriocarcinoma. After five cycles of EMA/ CO chemotherapy, his $\beta$-hCG levels decreased to normal. Three months later, his $\beta$-hCG levels increased, suggesting tumor recurrence. After four additional chemotherapy cycles, the patient died of progressive disease. Another 49-year-old man was diagnosed with retroperitoneal choriocarcinoma after the excision of a retroperitoneal mass. His $\beta$-hCG levels decreased to normal after 13 chemotherapy cycles. However, his $\beta$-hCG levels increased 18 months later. An additional ten cycles of chemotherapy were administered, but the patient died 54 months after the onset of disease.

In the literature cases, the median survival interval was 10 months (range, 6.4-13.6 months). The OS curves are shown in Figure 1. Univariate and multivariate analyses revealed that patients less than 34 years old (48 months versus [vs] 10 months; odds ratio [OR] $(95 \% \mathrm{CI})=0.47(0.27-0.81)$; $P=0.029)$, the presence of other histological components (54 months vs 11 months; OR $(95 \% \mathrm{CI})=0.54(0.32-0.92)$; $P=0.011$ ), and combination chemotherapy (14 months vs 2.5 months; OR $(95 \% \mathrm{CI})=0.18(0.06-0.53) ; P=0.002)$ were significantly associated with longer OS (Table 3 ).

\section{Discussion}

PCC, also known as nongestational choriocarcinoma, can occur in both men and women. Davidsohn ${ }^{7}$ described the first case of primary gastric choriocarcinoma in 1905. Most articles regarding this tumor have been case reports or small series, due to its rarity. The mechanism of PCC has long been debated, and no conclusions have been reached.

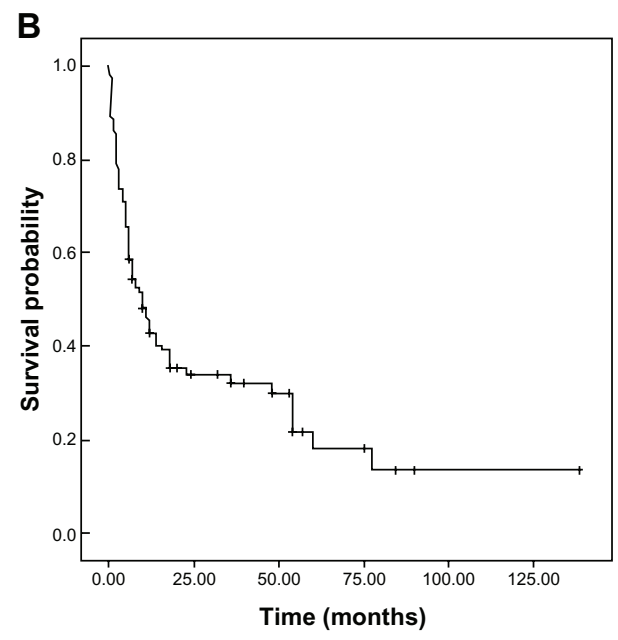


Table 3 Univariate and multivariate analyses for the OS periods (in months) with regard to males with PCC

\begin{tabular}{|c|c|c|c|c|c|c|c|}
\hline & \multirow[t]{2}{*}{$\mathbf{N}$} & \multirow{2}{*}{$\begin{array}{l}\text { I-year } \\
\text { survival (\%) }\end{array}$} & \multirow{2}{*}{$\begin{array}{l}\text { 3-year } \\
\text { survival (\%) }\end{array}$} & \multirow{2}{*}{$\begin{array}{l}\text { Median survival } \\
\text { interval (months) }\end{array}$} & \multirow{2}{*}{$\begin{array}{l}\text { Univariate } \\
P\end{array}$} & \multicolumn{2}{|c|}{ Multivariate } \\
\hline & & & & & & $P$ & OR $(95 \% \mathrm{Cl})$ \\
\hline \multicolumn{8}{|l|}{ Age } \\
\hline$<34$ years & 47 & 59.8 & 50.7 & 48 & 0.011 & 0.029 & $0.47(0.27-0.8 \mathrm{I})$ \\
\hline$\geq 34$ years & 52 & 38.6 & 24.8 & 10 & & & \\
\hline \multicolumn{8}{|l|}{ Histology } \\
\hline Coexisting with other & 22 & 68.6 & 53.5 & 54 & 0.090 & 0.011 & $0.54(0.32-0.92)$ \\
\hline Pure CC & 77 & 43.4 & 32.4 & 11 & & & \\
\hline \multicolumn{8}{|l|}{ Preoperative diagnosis } \\
\hline Yes & 35 & 41.4 & 20.7 & 10 & 0.063 & 0.023 & $1.84(1.09-3.10)$ \\
\hline No & 64 & 52.7 & 44.4 & 18 & & & \\
\hline \multicolumn{8}{|l|}{ Metastasis } \\
\hline Yes & 70 & 85.7 & 67.1 & 12 & 0.236 & & \\
\hline No & 29 & 65.5 & 49.1 & 36 & & & \\
\hline \multicolumn{8}{|l|}{ Chemotherapy } \\
\hline Yes & 94 & 50.6 & 38.5 & 14 & 0.001 & 0.002 & $0.18(0.06-0.53)$ \\
\hline No & 5 & 0 & 0 & 2.5 & & & \\
\hline \multicolumn{8}{|l|}{ Surgery } \\
\hline Yes & 37 & 50.1 & 39.5 & 10 & 0.761 & & \\
\hline No & 62 & 47.8 & 35.1 & 14 & & & \\
\hline
\end{tabular}

Abbreviations: OS, overall survival; PCC, primary choriocarcinoma; $\mathrm{CC}$, choriocarcinoma; $\mathrm{Cl}$, confidence interval.

Three hypotheses exist: 1) these tumors might arise from retained primordial germ cells that migrate abnormally during embryogenesis. ${ }^{8}$ This theory might explain the occurrence of carcinomas in midline structures such as the mediastinum, retroperitoneum, and the pineal gland; however, it cannot explain other choriocarcinoma locations such as the lung, liver, or stomach. 2) The tumor might be a testicular choriocarcinoma metastasis that regressed spontaneously. ${ }^{9}$ 3) The tumor might be a cancer that originally develops as a nontrophoblastic neoplasm and transforms into a choriocarcinoma. ${ }^{10}$ The latter theories seem more feasible with regard to explaining the occurrences of stomach and lung choriocarcinomas.

\section{Incidence of the disease}

Data regarding the gestational choriocarcinoma incidence rates are limited. Choriocarcinoma affects approximately one in 40,000 pregnancies in Europe and North America, whereas choriocarcinoma rates are higher in Southeast Asia and Japan (9.2 and 3.3 per 40,000 pregnancies, respectively). ${ }^{11}$ PCC is much rarer in men. According to the data collected at our hospital, the incidence ratio of PCC to gestational choriocarcinoma was 1:79. The male/female ratio was 13:33 among patients with PCC. The literature revealed that PCC is a rare disease that occurs at different sites. Less than $8 \%$ of testicular germ cell tumors contain a choriocarcinoma component, and pure choriocarcinoma accounts for only $0.3 \%$ of all primary testicular germ cell tumors. ${ }^{12}$ According to our data, males with PCC accounted for 3\% of all germ cell tumors after integrating the different types.

PCC can occur at different sites; however, the testis was the most common primary site at $36.2 \%(41 / 113)$, followed by the mediastinum (14/113), stomach (13/113), liver (12/113), lung (11/113), brain (8/113), small intestine $(6 / 113)$, and other sites (8/113). This disease can occur at different ages, from neonates to 80 years old; the median age found in the literature was 34 years old, compared with 31 years old among our cases. The peak age of occurrence was from 20 to 29 years, followed by 30 to 39 years. Patients in the former age group with suspicious symptoms should be checked for serum $\beta$-hCG to exclude PCC, because the disease rapidly progresses and can lead to early metastasis and death if undiagnosed. ${ }^{13}$ The peak age group in our results was similar to the results of Yokoi ${ }^{1}$ and Wang. ${ }^{1,14}$

\section{Diagnosis}

PCC can occur at different sites, across a wide age range. The lack of specific clinical symptoms and the common metastasis frequency (70/99) made preoperative diagnosis difficult. Diagnosis was possible when the site was near the surface of the skin and a biopsy could be performed relatively safely. However, biopsies cannot be performed at many sites (eg, the mediastinum, retroperitoneum, and pineal body). Even when biopsies can be performed, choriocarcinomas are not necessarily diagnosed easily. In a study by Kobayashi et al, only $8 \%$ of cases of primary gastric choriocarcinoma were 
diagnosed correctly via biopsy. ${ }^{15} \beta$-HCG has an important diagnostic value because PCC can also secrete $\beta$-hCG; thus, the proportion of patients with elevated serum $\beta$-hCG levels is high $(96.4 \%) .{ }^{1}$ However, serum $\beta-\mathrm{hCG}$ is almost never tested for among males. Only three cases were diagnosed preoperatively among those at $\mathrm{PUMCH}$, and these patients were all admitted to the hospital with masses at the primary site and elevated serum $\beta$-hCG levels. These three cases (Table 1) all died within 13 months after diagnosis. The investigation based on cases in the literature revealed that a preoperative diagnosis did not prolong the median overall survival ( 10 months vs 18 months, $P=0.063$ ). Thus, male PCC was difficult to diagnosis preoperatively, and a preoperative diagnosis did not change the prognosis.

\section{Treatment}

The investigation based on all 113 cases (the 13 PUMCH cases plus the 100 literature cases) revealed a poor prognosis. The median survival interval was 10 months. Of the PUMCH cases, six achieved long-term survival. The median survival interval was 54 months, which was much better than that reported in the literature. All 13 PUMCH patients (100\%) received surgery, whereas only 49 of 100 cases in the literature received surgery. The PUMCH patients received more chemotherapy cycles than those in the literature. Univariate and multivariate analyses revealed that patients no older than 34 years old, the presence of other histological components, and combination chemotherapy were significantly associated with longer survival intervals.

There is no standard management for males with PCC. Different treatment strategies were applied across the literature cases. These patients were treated with chemotherapy, radiotherapy, surgery, or some combination therein. An analysis of primary gastric choriocarcinoma found that synchronous liver metastasis, residual tumors after surgery, and the absence of chemotherapy significantly predicted reduced OS. ${ }^{15}$ Of the 13 PUMCH patients, four had no metastasis, three underwent radical resection (thereby achieving long survival intervals), and one underwent partial resection and died soon thereafter. Nine patients had metastasis: Two cases with primary and metastatic sites were totally resected (one of whom died 54 months after diagnosis, the other of whom survived). Regarding the corresponding outcomes of primary lung choriocarcinoma, we observed a slight tendency toward complete resection followed by chemotherapy or close follow-up examinations, thereby providing patients with the best likelihood of survival. ${ }^{16}$ The results of the PUMCH cases suggest that the complete resection of the primary site and metastases played important roles in the treatment of males with PCC. Since preoperative diagnosis does not prolong the median overall survival, early surgery without a diagnosis is still beneficial for overall survival.

Chemotherapy is a necessary treatment of gestational choriocarcinoma, ${ }^{17}$ even among males. ${ }^{18}$ Because most patients are diagnosed at an advanced stage of tumor metastasis, certain authors believe that all patients should receive chemotherapy. Adjuvant chemotherapy with two to four cycles of bleomycin, etoposide, and cisplatin (ie, PEB protocol) has been recommended for patients with ovarian choriocarcinomas. ${ }^{4,16}$ Most of the 13 PUMCH patients received chemotherapy with actinomycin D, etoposide, methotrexate, cyclophosphamide, and vincristine (ie, EMA/CO protocol); the median number of cycles was seven (range, 3-27 cycles).

Regular long-term clinical follow-up evaluations are important. Serum $\beta$-hCG levels should be used as a marker to assess treatment effectiveness ${ }^{19}$ and detect disease relapse. $\beta$-hCG levels decreased to normal ranges among the PUMCH patients who showed long-term survival and increased among relapsed PUMCH patients who died early. Relapse might denote poor prognosis because both relapsed patients died even after intensive chemotherapy had been administered.

There are some limitations to this study. This is a retrospective study, and bias could result from data collection. In addition, because of rarity of the disease, there is no reliable statistical analysis. Further studies are necessary.

\section{Conclusion}

PCC is an extremely rare disease in men. The age group of peak occurrence is 20 to 29 years old. The prognosis of PCC is poor, and no standard therapy exists. Most treatment options are empirical. Complete resection of the primary site and the metastases, even without preoperative diagnosis, combined with chemotherapy, improves the prognosis. Other prognostic factors include a patient age $<34$ years old or less, and the presence of other histological components.

\section{Disclosure}

The authors report no conflicts of interest in this work.

\section{References}

1. Yokoi K, Tanaka N, Furukawa K, et al. Male choriocarcinoma with metastasis to the jejunum: a case report and review of the literature. J Nippon Med Sch. 2008;75(2):116-121.

2. Lynch MJ, Blewitt GL. Choriocarcinoma arising in the male mediastinum. Thorax. 1953;8(2):157-161.

3. Shkolnik Z, Deutsch AA, Reiss R. Retroperitoneal choriocarcinoma in the male. Br J Urol. 1983;55(3):335-336.

4. Hadgu A, Tindni A, Panda M. Primary pulmonary choriocarcinoma in a male. BMJ Case Rep. 2010;2010: pii: bcr0220102712. 
5. Wildi-Runge S, Crevier L, Carret AS, Robitaille Y, Deal C. Pituitary choriocarcinoma in an adolescent male: tumor-derived $\mathrm{CG}$ and $\mathrm{GH}$ delay diagnosis. Growth Horm IGF Res. 2011;21(3):181-184.

6. Tokisue M, Yasutake K, Oya M, et al. Coexistence of choriocarcinoma and adenocarcinoma in the rectum: molecular aspects. J Gastroenterol. 1996;31(3):431-436.

7. Davidsohn C. Chorion-epitheliom and magenkrebs, eine seltene verschmelzung zweizer bosartiger geschwulste [Choriocarcinoma and gastric cancer: a rare mixture of two malignant tumors]. Charite Annal. 1905;29:426-436. German.

8. Rzeszutko M, Rzeszutko W, Nienartowicz E, Jeleń M. Paratesticular localization of burned out non-seminomatous germ cell tumor NSGCT: a case report. Pol J Pathol. 2006;57(1):55-57.

9. Fine G, Smith RW, Pachter MR. Primary extragenital choriocarcinoma in the male subject. Case report and review of the literature. Am J Med. 1962;32:776-794.

10. Toda $\mathrm{S}$, Inoue $\mathrm{Y}$, Ishino $\mathrm{T}$, et al. A rare case of primary pulmonary choriocarcinoma in a male: immunohistochemical detection for human chorionic gonadotropin, epidermal growth factor (EGF) and EGFreceptor. Endocr J. 1995;42(5):655-659.

11. Lurain JR. Gestational trophoblastic disease I: epidemiology, pathology, clinical presentation and diagnosis of gestational trophoblastic disease, and management of hydatidiform mole. Am J Obstet Gynecol. 2010;203(6):531-539.
12. Ohr J. Tumors of the testis, adnexa, spermatic cord, and scrotum. Arch Pathol Lab Med. 2000;124(12):1855.

13. Syed S, Westwood AJ. Clinical reasoning: a 25-year-old man with headaches and collapse. Neurology. 2013;80(20):e211-e214.

14. Wang L, Pitman MB, Castillo CF, Dal Cin P, Oliva E. Choriocarcinoma involving the pancreas as first manifestation of a metastatic regressing mixed testicular germ cell tumor. Mod Pathol. 2004;17(12): 1573-1580.

15. Kobayashi A, Hasebe T, Endo Y, et al. Primary gastric choriocarcinoma: two case reports and a pooled analysis of 53 cases. Gastric Cancer. 2005;8(3):178-185.

16. Serno J, Zeppernick F, Jäkel J, et al. Primary pulmonary choriocarcinoma: case report and review of the literature. Gynecol Obstet Invest. 2012; 74(2):171-176.

17. Lurain JR. Gestational trophoblastic disease II: classification and management of gestational trophoblastic neoplasia. Am J Obstet Gynecol. 2011;204(1):11-18.

18. Noguchi T, Takeno S, Sato T, Takahashi Y, Uchida Y, Yokoyama S. A patient with primary gastric choriocarcinoma who received a correct preoperative diagnosis and achieved prolonged survival. Gastric Cancer. 2002;5(2):112-117.

19. Jiang L, Wu JT, Peng X. Primary choriocarcinoma of the colon: a case report and review of the literature. World J Surg Oncol. 2013;11:23.
OncoTargets and Therapy

\section{Publish your work in this journal}

OncoTargets and Therapy is an international, peer-reviewed, open access journal focusing on the pathological basis of all cancers, potential targets for therapy and treatment protocols employed to improve the management of cancer patients. The journal also focuses on the impact of management programs and new therapeutic agents and protocols on

\section{Dovepress}

patient perspectives such as quality of life, adherence and satisfaction. The manuscript management system is completely online and includes a very quick and fair peer-review system, which is all easy to use. Visit http://www.dovepress.com/testimonials.php to read real quotes from published authors. 